\title{
BIBLIOTECAS UNIVERSITÁRIAS E A ACESSIBILIDADE AOS USUÁRIOS SURDOS E COM DEFICIÊNCIA AUDITIVA
}

\author{
LAS BIBLIOTECAS UNIVERSITARIAS Y LA \\ ACCESIBILIDAD PARA LOS USUARIOS CON \\ DISCAPACIDADES SORDOS Y OYENTES
}

\author{
Sulamita Nicolau de Miranda - sulamitandmiranda@gmail.com \\ Mestra em Biblioteconomia pelo PPGB/UNIRIO. Bibliotecária- \\ Documentalista do IFCS/UFRJ \\ Marcos Luiz Cavalcanti de Miranda-mlmiranda@unirio.br \\ Doutor em Ciência da Informação pelo Instituto Brasileiro de \\ Informação em Cîência e Tecnologia (IBICT/UFRJ). Professor \\ Associado do PPGB/UNIRIO.
}

\begin{abstract}
RESUMO
Introdução: Aborda a questão da acessibilidade aos usuários surdos e com deficiência auditiva em bibliotecas universitárias analisando o Sistema de Bibliotecas da Universidade Federal do Estado do Rio de Janeiro UNIRIO/UNIBIBLI.

Objetivos: verificar a adoção das recomendações das Diretrizes para Serviços de Biblioteca para Surdos (DSBS) da IFLA pelas bibliotecas do Sistema UNIBIBLI; identificar quais itens recomendados pelas DSBS são adotados pelas bibliotecas do UNBIBLI para garantir o atendimento aos usuários surdos e com deficiência auditiva; verificar se o UNIBIBLI tem uma política de acessibilidade que atenda às necessidades dos usuários surdos e com deficiência auditiva; identificar quais os parâmetros de acessibilidade adotados pelas bibliotecas analisadas no atendimento aos usuários surdos e com deficiência auditiva.

Metodologia: Adota-se a metodologia exploratória com abordagem qualitativa, os instrumentos de coleta de dados utilizados foram o questionário e a entrevista.

Resultados: Apontam que o UNIBIBLI não tem uma política de acessibilidade que atenda as especificidades dos usuários surdos e com deficiência auditiva, e que o Sistema adota parcialmente os itens recomendados pelas Diretrizes para Serviços de Bibliotecas para Surdos da IFLA, mas manifestou interesse em adequar-se às referidas recomendações.
\end{abstract}


Conclusões: A questão da acessibilidade não pode ser encarada apenas como o cumprimento da legislação e normas técnicas, é imprescindível que os profissionais que atuam nas bibliotecas estejam cientes de seus papéis perante a sociedade e da função social da biblioteca.

Palavras-chave: Bibliotecas universitárias. Acessibilidade. Surdos. Deficiência auditiva. Diretrizes para Serviços de Bibliotecas para Surdos. Federação Internacional das Associações e Instituições Bibliotecárias.

\section{INTRODUÇÃO}

A questão da inclusão social das pessoas com deficiência tem sido objeto de apreciação por diversos setores da sociedade em virtude do engajamento e das constantes lutas do movimento das pessoas com deficiência, que tem resultado em legislação nacional e internacional com o intuito de assegurar a essas pessoas seus direitos.

No âmbito da Biblioteconomia essa temática tem sido abordada desde longa data. Ranganathan já tratava essa questão em 1931 em seu livro "As Cinco Leis da Biblioteconomia" e preocupava-se em disseminar que os livros eram para todas as pessoas independentemente das necessidades especiais que apresentassem.

Na segunda lei de Ranganathan - para cada leitor seu livro observa-se que o intuito é permitir o acesso à informação a todos os indivíduos de forma igualitária, todos terão a mesma oportunidade de ter acesso aos livros esteja este em Braille, audiolivro, DVD legendado, DVD em Libras ou qualquer outro suporte:

[...] No entanto a Segunda Lei tratará a todos como iguais e oferecerá a CADA UM O SEU LIVRO. Obedecerá escrupulosamente ao princípio da igualdade de oportunidades em relação aos livros, ao ensino e ao entretenimento. (RANGANATHAN, 2009, p. 92).

Esse entendimento de Ranganathan demonstra o compromisso da Biblioteconomia em garantir ao usuário com deficiência o seu direito de acesso à informação. 
Especificamente em relação às pessoas surdas e com deficiência auditiva os dados do Censo 2010 do Instituto Brasileiro de Geografia e Estatística - IBGE ${ }^{1}$ informam que cerca de 9,7 milhões de pessoas declararam-se deficientes auditivas, o que representa $5,1 \%$ da população brasileira. Dentre essas pessoas 344,2 mil são surdas e 1,7 milhão de pessoas têm grande dificuldade de ouvir.

No âmbito das Universidades verifica-se o desenvolvimento do número de alunos surdos e com deficiência auditiva matriculados nas Instituições de Ensino Superior, públicas e privadas, nos últimos anos de acordo com dados do Instituto Nacional de Estudos e Pesquisas Educacionais - INEP': em 2011 encontravam-se matriculados 1.582 alunos surdos e 4.078 com deficiência auditiva, em 2012 eram 1.650 surdos e 6.008 com deficiência auditiva e em 2013 eram 1.488 alunos surdos e 7.037 com deficiência auditiva.

Esses dados nos levam a refletir se as bibliotecas universitárias estão preparadas ou se preparando para atender essa demanda e nesse sentido realizamos uma avaliação das condições de acessibilidade aos usuários surdos e com deficiência auditiva no Sistema de Bibliotecas da Universidade Federal do Estado do Rio de Janeiro - UNIRIO/UNIBIBLI, tendo como documento norteador da pesquisa as Diretrizes para Serviços de Bibliotecas para Surdos da Federação Internacional de Associações e Instituições Bibliotecárias IFLA. Nesse trabalho as referidas diretrizes serão denominamos de DSBS.

A pesquisa teve como objetivo geral verificar a adoção das recomendações das Diretrizes para Serviços de Biblioteca para Surdos da IFLA pelas bibliotecas do Sistema UNIBIBLI da Universidade Federal do Estado do Rio de Janeiro - UNIRIO e os objetivos específicos foram: Identificar quais itens de acessibilidade, recomendados pelas DSBS são

\footnotetext{
${ }^{1}$ G1 Globo.com (2012).

2 INEP (2011).
} 
adotados pelas bibliotecas do Sistema de Bibliotecas da UNIRIO para garantir o atendimento aos usuários surdos e com deficiência auditiva; Verificar se o Sistema de Bibliotecas da UNIRIO tem uma política de acessibilidade que atenda às necessidades dos usuários surdos e com deficiência auditiva; Identificar quais os parâmetros de acessibilidade adotados pelas bibliotecas analisadas no atendimento aos usuários surdos e com deficiência auditiva.

Esse artigo apresenta os resultados obtidos na pesquisa de mestrado apresentado ao Programa de Pós-Graduação em Biblioteconomia da UNIRIO.

\section{BIBLIOTECAS UNIVERSITÁRIAS, ACESSO À INFORMAÇÃO E ACESSIBILIDADE}

O direito de acesso à informação está assegurado pela Constituição brasileira e é um elemento para o desenvolvimento da cidadania, uma vez que munido de conhecimento o cidadão poderá participar mais ativamente da sociedade, atuando com autonomia e crítica.

O termo acessibilidade é adotado em diversas normas, leis e decretos e podemos observar a presença da palavra autonomia nesses conceitos legais, dos quais destacamos.

Para a Lei $n^{\circ}$ 10.098, de 19 de dezembro de 2000 acessibilidade é a:

Art. $2^{\circ}, \mathrm{I}[\ldots]$ possibilidade e condição de alcance para utilização, com segurança e autonomia, dos espaços, mobiliários e equipamentos urbanos, das edificações, dos transportes e dos sistemas e meios de comunicação, por pessoa portadora de deficiência [sic] ou com mobilidade reduzida. (BRASIL, 2000, grifo nosso).

O decreto $n^{\circ}$ 5.296, de 02 de dezembro de 2004 define acessibilidade como a: 
Art. 8ำ I [...] condição para utilização, com segurança e autonomia, total ou assistida, dos espaços, mobiliários e equipamentos urbanos, das edificações, dos serviços de transporte e dos dispositivos, sistemas e meios de comunicação e informação, por pessoa portadora de deficiência $[\mathrm{sic}]^{3}$ ou com mobilidade reduzida [...]. (BRASIL, 2004, grifo nosso).

Para a Norma ABNT NBR 9050/15 acessibilidade é a:

possibilidade e condição de alcance, percepção e entendimento para utilização, com segurança e autonomia, de espaços, mobiliários, equipamentos urbanos, edificações, transportes, informação e comunicação, inclusive seus sistemas e tecnologias, bem como outros serviços e instalações abertos ao público, de uso público ou privado de uso coletivo, tanto na zona urbana como na rural, por pessoa com deficiência ou mobilidade reduzida. (ABNT, 2015, p. 2, grifo nosso).

O termo autonomia presente nos conceitos de acessibilidade indicados acima, demonstra que precisamos concentrar nossos esforços para adequar os serviços, produtos e espaços das bibliotecas para que os usuários com deficiência possam usufruir de forma plena do ambiente da biblioteca.

Câmara (2011, p. 6) enfatiza o papel social da biblioteca afirmando que esta deve: "[...] contribuir para o social, educacional e cultural, dando a oportunidade a todos sem restrições, o direito de utilizar do seu acervo o conhecimento desejado."

Nesse contexto, a biblioteca universitária visa prover à comunidade acadêmica acesso à informação contribuindo com o ensino, a extensão e a pesquisa gerando a produção de novos conhecimentos que trarão benefícios para toda a sociedade.

Ferreira (1980, p.[1]) nos esclarece que "[...] não cabe à educação meramente transmitir conhecimentos, experiências e ideais,

\footnotetext{
${ }^{3}$ O termo adotado hoje é pessoa com deficiência. Nesse artigo utilizaremos a expressão sic
} após todos os termos que não seguirem essa terminologia. 
mas, sobretudo, dar a instrumentação necessária para que cada um, por si mesmo, seja capaz de fazer descobertas, criar e construir algo."

Essa afirmativa se coaduna com o conceito de acessibilidade, trazido pela Lei ㄲo 10.098/00 - art.2o, I, pelo Decreto № 5.296/04 art.8ํㅡ, I, e pela norma NBR 9050/15 que visa oferecer autonomia à pessoa com deficiência para que esta possa realizar suas próprias atividades, conforme supracitado.

A importância da acessibilidade no âmbito universitário foi reconhecida e tornou-se um dos requisitos para autorização, reconhecimento de cursos e de credenciamento de instituições, conforme Portaria no 3.284/03 do Ministério da Educação, tendo como intuito assegurar às pessoas com deficiência "condições básicas de acesso ao ensino superior, de mobilidade e de utilização de equipamentos e instalações das instituições de ensino".

O Decreto $n^{\circ} 5.296 / 04$ estabelece que a universidade deve proporcionar condições de acesso e utilização da biblioteca pelas pessoas com deficiência:

Art. 24. Os estabelecimentos de ensino de qualquer nível, etapa ou modalidade, públicos ou privados, proporcionarão condições de acesso e utilização de todos os seus ambientes ou compartimentos para pessoas portadoras de deficiência [sic] ou com mobilidade reduzida, inclusive salas de aula, bibliotecas, auditórios, ginásios e instalações desportivas, laboratórios, áreas de lazer e sanitários. (BRASIL, 2004, grifo nosso).

Tornar as bibliotecas universitárias um ambiente democrático, acessível a todos é um desafio para o qual os bibliotecários e as bibliotecas precisam se preparar. Especificamente no que se refere ao atendimento ao usuário surdo e com deficiência auditiva além da legislação nacional existem as Diretrizes para Serviços de Bibliotecas para Surdos da IFLA que irão contribuir para o estabelecimento de produtos e serviços compatíveis com as necessidades informacionais desses usuários como apresentaremos na próxima seção. 


\section{DIRETRIZES PARA SERVIÇOS DE BIBLIOTECAS PARA SURDOS}

Vimos que $5,1 \%$ da população brasileira é surda ou possui deficiência auditiva e que o número de alunos surdos e com deficiência auditiva matriculados nas instituições de ensino superior tem se desenvolvido consideravelmente.

Nos termos do art. 2o do Decreto № 5.626/05 (BRASIL, 2005) é considerada surda a pessoa que "por ter perda auditiva, compreende e interage com o mundo por meio de experiências visuais, manifestando sua cultura principalmente pelo uso da Língua Brasileira de Sinais Libras".

Ainda de acordo com o referido decreto a deficiência auditiva é a "perda bilateral, parcial ou total, de quarenta e um decibéis $(\mathrm{dB})$ ou mais, aferida por audiograma nas frequências de $500 \mathrm{~Hz}, 1.000 \mathrm{~Hz}, 2.000 \mathrm{~Hz}$ e $3.000 \mathrm{~Hz}$.

Pensando nessas pessoas e em suas necessidades específicas, a Federação Internacional de Associações e Instituições Bibliotecárias IFLA elaborou o documento intitulado "Diretrizes para Serviços de Biblioteca para Surdos" - "Guidelines for Library Services to Deaf People".

Esse documento, conforme informamos na introdução, será denominado nesse trabalho de DSBS.

As diretrizes são um instrumento que visa nortear os serviços oferecidos aos usuários surdos e com deficiência auditiva em todos os tipos de biblioteca, para que essas possam se adaptar e oferecer um atendimento mais adequado a esses usuários e foram endossadas pela Federação Mundial de Surdos - World Federation of the Deaf.

As Diretrizes para Serviços de Bibliotecas para Surdos foram elaboradas "[...] com o intuito de informar bibliotecários sobre as necessidades do surdo em relação às bibliotecas e à informação, e pertencem a todas as bibliotecas que têm surdos como uma parcela de seus usuários." (IFLA, 2000, p. 7). 
Têm por propósito: "[...] servir de estímulo para estabelecer serviços para surdos e como instrumento para avaliação da completude e da qualidade desses serviços [...]" (IFLA, 2000, p. 7).

As DSBS afirmam que as bibliotecas "[...] tem a responsabilidade de garantir que suas coleções e serviços sejam acessíveis aos surdos e que os surdos estejam cientes dos serviços que as bibliotecas podem Ihes prover" (IFLA, 2000, p. 6).

Em nossa pesquisa utilizamos a $2^{2}$ edição de 2000 na versão traduzida para o português por Ana Maria V. C. Duckworth e prefácio para a edição brasileira de Leland Emerson McCleary - Universidade de São Paulo (USP).

As DSBS estão divididas em cinco categorias: Pessoal, Comunicação, Acervo, Serviços e Divulgação dos programas. Cada uma dessas categorias apresenta uma série de direcionamentos para atender às necessidades desses usuários.

Para que possamos compreender a relevância desse documento apresentaremos a seguir, resumidamente, algumas recomendações de cada uma das cinco categorias indicadas acima.

A categoria Pessoal apresenta recomendações quanto à capacitação profissional dos funcionários da biblioteca quanto às necessidades especiais dos usuários surdos, por exemplo, necessidades de comunicação, cultura surda, coleções de materiais especiais etc., para que possam ser elaborados produtos e serviços que atendam as necessidades desses usuários.

Outro aspecto que destacamos nessa categoria é o papel das instituições de ensino na área da graduação em Biblioteconomia de oferecer disciplinas voltadas para as questões das pessoas com deficiência, da inclusão social para que o profissional entre em contato com essa temática desde sua formação na graduação.

$\mathrm{Na}$ categoria Comunicação ressaltamos a importância de toda a equipe da biblioteca receber treinamento em como comunicar-se com os surdos, pois a comunicação entre surdos e ouvintes ocorre de várias 
maneiras dependendo do "[...] grau de perda auditiva, identidade cultural e do contexto, surdos podem utilizar a língua de sinais, a fala, a escrita, gestos, ou qualquer combinação desses meios." (IFLA, 2000, p. 10).

Outra recomendação dessa categoria é a necessidade de manter a comunicação pela internet acessível aos usuários surdos, ou seja, os sites, blogs, redes sociais etc. precisam estar acessíveis para esses usuários.

Na categoria Acervo o destaque é a necessidade de compor o acervo com DVDs legendados ou em língua de sinais e também de disponibilizar materiais relacionados à surdez e à cultura surda.

$\mathrm{Na}$ categoria Serviços a recomendação é que "[...] todos os acervos, serviços e programas da biblioteca devem ser acessíveis a sua comunidade surda". Por exemplo, os treinamentos de usuários devem ser oferecidos também com a opção de interpretação em língua de sinais. (IFLA, 2000, p. 15).

Já na categoria Divulgação dos programas destacamos a necessidade de intensificar a divulgação dos serviços acessíveis oferecidos pela biblioteca, de forma a atrair esse usuário a utilizar a biblioteca.

A partir desse documento, dividido nas cinco categorias elencadas acima, elaboramos a pergunta problema da nossa pesquisa: A política de acessibilidade do Sistema de Bibliotecas da Universidade Federal do Estado do Rio de Janeiro - UNIBIBLI/UNIRIO adota as recomendações das DSBS da IFLA no atendimento aos usuários surdos e com deficiência auditiva?

E para responder a essa pergunta traçamos como objetivo geral verificar a adoção das recomendações das Diretrizes para Serviços de Biblioteca para Surdos da IFLA pelas bibliotecas do Sistema UNIBIBLI da Universidade Federal do Estado do Rio de Janeiro - UNIRIO e os objetivos específicos são: Identificar quais itens de acessibilidade, recomendados pelas DSBS são adotados pelas bibliotecas do Sistema de Bibliotecas da UNIRIO para garantir 0 atendimento aos usuários 
surdos e com deficiência auditiva; Verificar se o Sistema de Bibliotecas da UNIRIO tem uma política de acessibilidade que atenda às necessidades dos usuários surdos e com deficiência auditiva; Identificar quais os parâmetros de acessibilidade adotados pelas bibliotecas analisadas no atendimento aos usuários surdos e com deficiência auditiva.

\section{PROCEDIMENTOS METODOLÓGICOS}

Trata-se de uma pesquisa exploratória com abordagem qualitativa, com apoio na pesquisa bibliográfica e documental, sendo utilizados como instrumentos de coleta de dados o questionário e entrevista estruturada com os gestores das bibliotecas e diretoria do Sistema UNIBIBLI respectivamente.

Esse estudo fundamenta-se na Segunda Lei de Ranganathan - A cada leitor seu livro; nas Diretrizes de Serviços para Bibliotecas para Surdos, uma vez que apresentam recomendações específicas de serviços de bibliotecas para as pessoas surdas e foi endossada pela Federação Mundial dos Surdos; nos quesitos de acessibilidade apresentados por Sassaki (apud VIVARTA, 2003): acessibilidade arquitetônica, acessibilidade comunicacional, acessibilidade metodológica, acessibilidade instrumental, acessibilidade programática e acessibilidade atitudinal e na promoção da inclusão e da erradicação da discriminação previstos no Código de Ética da IFLA para Bibliotecários e outros Profissionais da Informação (IFLA, 2012).

O Sistema de Bibliotecas da UNIRIO - UNIBIBLI - constitui o universo da pesquisa e é composto pelas: Biblioteca Central; Biblioteca Setorial do Centro de Ciências Humanas e Sociais $(\mathrm{CCH})$; Biblioteca Setorial do Centro de Letras e Artes (CLA); Biblioteca Setorial da Escola de Medicina e Cirurgia (CCBS/URM); Biblioteca Setorial do Instituto Biomédico (CCBS/URB); Biblioteca Setorial de Enfermagem e Nutrição 
(CCBS/URS);Biblioteca Setorial do Centro de Ciências Jurídicas e Políticas (CCJP).

Elaboramos um questionário composto por 28 perguntas, das quais 27 foram baseadas nas DSBS e uma pergunta aberta sobre os parâmetros de acessibilidade adotados pelo Sistema UNIBIBLI para esses usuários. As perguntas do questionário foram divididas em cinco categorias de acordo com as DSBS: Pessoal, Comunicação, Acervo, Serviços e Divulgação dos programas.

Utilizamos uma pergunta com o intuito de verificar se o UNIBIBLI utilizava algum outro padrão para garantir a acessibilidade aos usuários surdos e com deficiência auditiva diferente dos recomendados pelas DSBS da IFLA, pois poderia não seguir essas recomendações, mas ter uma política de acessibilidade que contemplasse esses usuários.

A diretoria do Sistema respondeu ao roteiro de entrevista com três perguntas abertas com a finalidade de identificar se o Sistema tinha uma política de acessibilidade e se essa política atendia as necessidades dos usuários surdos e com deficiência auditiva.

\section{ANÁLISE DOS RESULTADOS}

Considerando que o objetivo geral da pesquisa consistia em verificar se o Sistema de Bibliotecas da UNIRIO - UNIIBIBLI adotava as recomendações das Diretrizes para Serviços de Bibliotecas para Surdos da IFLA, os resultados apontaram que o UNIBIBLI adota parcialmente alguns itens recomendados pelas DSBS e não dispõe de uma política de acessibilidade que atenda as especificidades dos usuários surdos e com deficiência auditiva, mas demonstrou interesse em adequar-se e melhorar as condições de acessibilidade a esses usuários.

Os resultados da pesquisa demonstraram que as bibliotecas que compõem o Sistema UNIBIBLI/UNIRIO ainda não dispõem de uma política de acessibilidade que atenda às necessidades dos usuários surdos e com deficiência auditiva, mas algumas bibliotecas do Sistema já adotam parcialmente as recomendações das DSBS. 
O UNIBIBLI ao aceitar participar dessa pesquisa, permitindo um diagnóstico das condições de acessibilidade aos usuários surdos e com deficiência auditiva colocou em prática a acessibilidade atitudinal buscando conhecer as necessidades desses usuários e se propondo a melhorar a forma de atendê-los sem preconceitos ou estigmas, apenas atendendo suas necessidades.

\section{CONSIDERAÇÕES FINAIS}

A biblioteca universitária exerce um papel importante contribuindo para a geração de conhecimento e o desenvolvimento da pesquisa nas universidades que irão refletir em toda a sociedade como produtos, serviços e novos conhecimentos que beneficiarão a todos. Nesse sentido, precisa estar acessível para toda a comunidade acadêmica, independentemente das diversidades que nela existam.

Para que o Sistema UNIBIBLI possa efetuar as adequações necessárias ressaltamos a importância de realizar parcerias com outros setores da Universidade, porque essas adequações requerem o apoio de uma equipe multidisciplinar, por exemplo: com intérprete de Libras, profissionais de tecnologia da informação, setor de recursos financeiros, setor de pessoal etc.

Cabe lembrar ainda, a necessidade de buscar apoio nas experiências de outras instituições. O que tem sido feito, como tem sido feito e o que pode ser aproveitado e viabilizado pelo Sistema UNIBIBLI, além de estar atento aos avanços tecnológicos que tanto tem contribuído para possibilitar a comunicação entre as pessoas surdas e com deficiência auditiva.

Sendo assim, esperamos com essa pesquisa que o UNIBIBLI execute as ações necessárias para a elaboração de uma política de acessibilidade compatível com as necessidades dos usuários surdos e com deficiência auditiva, e que as recomendações já adotadas parcialmente sejam estendidas a todas as bibliotecas do Sistema. 
Ressaltamos que a questão da acessibilidade não pode ser encarada apenas como o cumprimento da legislação e normas técnicas, é imprescindível que os profissionais que atuam nas bibliotecas estejam cientes de seus papéis perante a sociedade e da função social da biblioteca. Precisam estar atentos para compreender e respeitar as peculiaridades dos usuários com deficiência para adequar os serviços e produtos, bem como melhor atendê-los, garantindo-Ihes não somente o acesso à informação, mas abrindo espaço para que exerçam sua cidadania e tornando a biblioteca um ambiente agradável de acolhimento para estudo e lazer.

\section{REFERÊNCIAS}

ASSOCIAÇÃO BRASILEIRA DE NORMAS TÉCNICAS. NBR 9050. Acessibilidade a edificações, espaço, mobiliário e equipamentos urbanos. 3.ed. Rio de Janeiro: ABNT, 2015.

BRASIL. Lei no 10.098, de 19 de dezembro de 2000. Estabelece normas gerais e critérios básicos para a promoção da acessibilidade das pessoas portadoras de deficiência ou com mobilidade reduzida, e dá outras providências. 2000. Disponível em:<http://www.planalto.gov.br/ccivil_03/leis/10098.htm>. Acesso em: 03 mar. 2014.

. Decreto $\mathrm{n}$ - 5.296, de 2 de dezembro de 2004. Regulamenta as Leis $n^{\circ \mathrm{S}} 10.048$, de 8 de novembro de 2000 , que dá prioridade de atendimento às pessoas que especifica, e 10.098, de 19 de dezembro de 2000, que estabelece normas gerais e critérios básicos para a promoção da acessibilidade das pessoas portadoras de deficiência ou com mobilidade reduzida, e dá outras providências. 2004. Disponível em: <http://www.planalto.gov.br/ccivil_03/_ato20042006/2004/decreto/d5296.htm>. Acesso em: 27 mar. 2014.

Decreto $\mathrm{n}^{\circ}$ 5.626, de 22 de dezembro de 2005. Regulamenta a Lei № 10.436 , de 24 de abril de 2002, que dispõe sobre a Língua Brasileira de Sinais - Libras, e o art. 18 da Lei № 10.098, de 19 de dezembro de 2000. 2005. Disponível em:<http://www.libras.org.br>. Acesso em: 20 nov. 2013. 
Ministério da Educação. Portaria no 3.284, de 7 de novembro

de 2003. Dispõe sobre requisitos de acessibilidade de pessoas

portadoras de deficiências, para instruir os processos de autorização e de reconhecimento de cursos, e de credenciamento de instituições.

Disponível em:

$<$ http://portal.mec.gov.br/seesp/arquivos/pdf/port3284.pdf>. Acesso em 20 ago. 2015.

FERREIRA, Lusimar Silva. Bibliotecas universitárias brasileiras. São Paulo: Livraria Pioneira, 1980.

G1 Globo.com. 23,9\% dos brasileiros declaram ter alguma deficiência, diz IBGE. Disponível em:

<http://g1.globo.com/brasil/noticia/2012/04/239-dos-brasileirosdeclaram-ter-alguma-deficiencia-diz-ibge.html>. Acesso em: 20 ago. 2014.

INSTITUTO NACIONAL DE ESTUDOS E PESQUISAS EDUCACIONAIS ANÍSIO TEIXEIRA - INEP. Sinopses estatísticas da educação

superior. Disponível em: <http://portal.inep.gov.br/superiorcensosuperior-sinopse>. Acesso em: 20 ago. 2014.

INTERNATIONAL FEDERATION OF LIBRARY ASSOCIATIONS AND INSTITUTTIONS. Diretrizes para serviços de biblioteca para surdos. Editado por John Michael Day; tradução Ana Maria V. C. Duckworth. Prefácio para a edição brasileira Leland Emerson McCleary. 2.ed. 2000. Série Publicações Ocasionais, No. 1. São Paulo, A Escola do Futuro, Universidade de São Paulo. (IFLA Professional Reports: 62). Disponível em: <http://especial.futuro.usp.br/documentos/guiaifla.rtf>. Acesso em: 29 mar. 2014

Código de ética da IFLA para bibliotecários e outros profissionais da informação. 2012. Disponível em: <http://www. ifla.org/files/assets/faife/codesofethics/portuguesecodeofethi csfull.pdf>. Acesso em: 22 jun. 2014.

NASCIMENTO, Geysa Flávia Câmara de Lima. O bibliotecário de referência e o usuário portador de necessidade auditiva: um olhar sobre a prática. In: CONGRESSO BRASILEIRO DE BIBLIOTECONOMIA, DOCUMENTAÇÃO E CIÊNCIA DA INFORMAÇÃO, 24., 2011, Maceió. Anais eletrônicos... Maceió, 2011. Disponível em: $<w w w . f e b a b . o r g . b r / c o n g r e s s o s / i n d e x . p h p / c b b d / x x i v / p a p e r / . . / 608 / 375>$. Acesso em: 24 jul. 2013.

RANGANATHAN, S.R. As cinco leis da biblioteconomia. Brasília: Briquet de Lemos, 2009. 
VIVARTA, Veet. (Coord.). Mídia e deficiência. Brasília : ANDI ; Fundação Banco do Brasil, 2003. (Série Diversidade). Disponível em: <http://www.andi.org.br/sites/default/files/Midia_e_deficiencia.pdf $>$. Acesso em: 15 jun. 2014.

\section{Title}

University libraries and accessibility for deaf and hard of hearing users

\section{Abstract}

Introduction: The study analyzes the Library Systems of the Federal University of the State of Rio de Janeiro (UNIRIO/UNIBIBLI) as far as access to university library services by deaf and hard of hearing users is concerned.

Objectives: To verify the adoption of the recommendations of the Guidelines for Library Services to Deaf (DSB) IFLA by the UNIBIBLI system libraries; identify which items recommended by DSB are adopted by UNBIBLI libraries to ensure service to the deaf and hard of hearing users; verify that the UNIBIBLI has an accessibility policy that meets the needs of the deaf and hard of hearing users; identify the accessibility parameters adopted by libraries analyzed in serving deaf and hard of hearing users.

Methodology: It adopts to exploratory methodology with a qualitative approach, the data collection instruments used were the questionnaire and the interview.

Results: The results show that UNIBIBLI does not have an accessibility policy that meets the needs of deaf and hard of hearing users and that the System partially follows recommendations laid out by IFLA Guidelines for Library Services to Deaf People, but demonstrated interest in alignment with the said recommendations.

Conclusions: The issue of accessibility can not be seen only as the fulfillment of the legislation and technical standards, it is essential that professionals who work in libraries are aware of their roles in society and library of social function.

Keywords: University libraries. Accessibility. Deaf. Hard of heraring. Guidelines for Library Services to Deaf People. International Federation of Library Association and Institutions.

\section{Titulo}

Las bibliotecas universitarias y la accesibilidad para los usuarios con discapacidades sordos y oyentes

\section{Resumen}

Introducción: Se aborda el tema de la accesibilidad a las personas sordas y con problemas de audición en las bibliotecas universitarias analizando el 
Sistema de Bibliotecas de la Universidad Federal del Estado de Río de Janeiro - UNIRIO/UNIBIBLI.

Objectivos: Comprobar la adopción de las recomendaciones de la Guía para los Servicios Bibliotecarios para Personas Sordas (DSB) IFLA por las bibliotecas del sistema UNIBIBLI; identificar los elementos recomendados por DSB son adoptadas por las bibliotecas UNBIBLI para garantizar el servicio a las personas sordas y con problemas de la audición; verificar que el UNIBIBLI tiene una política de accesibilidad que cumpla con las necesidades de las personas sordas y con problemas de la audición; identificar los parámetros de accesibilidad adoptadas por las bibliotecas analizadas en el servicio a personas sordas o con problemas de de la audición.

Metodología: Adopta a metodología exploratoria con un enfoque cualitativo, los instrumentos de recolección de datos utilizados fueron el cuestionario y la entrevista.

Resultados: Se apuntan al UNIBIBLI tiene una política de accesibilidad que se ocupa de las características específicas de las personas sordas y con problemas de la audición, y el sistema adopta parcialmente los elementos recomendados por la Guía para los Servicios Bibliotecarios para Personas Sordas de la IFLA, pero expresaron su interés en adecuar hasta las Recomendaciones.

Conclusiones: El tema de la accesibilidad no puede ser visto sólo como el cumplimiento de la legislación y las normas técnicas, es esencial que los profesionales que trabajan en las bibliotecas son conscientes de su papel en la sociedad y la biblioteca de la función social.

Palabras clave: Las bibliotecas universitarias. Accesibilidad. Sordos. Pérdida de audición. Guía para servicios de biblioteca para los Sordos. Federación Internacional de Asociaciones de Bibliotecarios y Bibliotecas.

Recebido em: 10.10 .2015

Aceito em: 20.12.2015 